\title{
MOYA MOYA: SPECTRUM OF IMAGING
}

Jai Kishore' ${ }^{1}$ Ramkumar², Bulabai Karpagam³

\section{HOW TO CITE THIS ARTICLE:}

Jai Kishore, Ramkumar, Bulabai Karpagam. "Moya Moya: Spectrum of Imaging”. Journal of Evolution of Medical and Dental Sciences 2014; Vol. 3, Issue 60, Nov 10; Page: 13469-13472, DOI: 10.14260/jemds/2014/3795

CLINICAL SUMMARY: This article is a case report of a 6 YR old child who was admitted with c/o headache on \& off for past 3 yrs associated with h/o drowsiness \& vomiting. h/o drop attacks present. no h/o trauma.

DIFFERENTIAL DIAGNOSIS: Moyamoya syndrome (also termed the moyamoya pattern or phenomenon) is due to numerous conditions that can cause arterial occlusion of the circle of Willis, with resultant collaterals, and appearances reminiscent of moyamoya disease. These conditions include:

Cranial radiotherapy

Neurofibromatosis type 1 (NF1)

Tuberous sclerosis (TS)

Infection Auto immune disorders

sickle cell disease, $\bullet$ Fanconi anaemia, aplastc anaemia

Down syndrome, Apert syndrome

Oral contraceptive use

Atherosclerosis

Final Diagnosis: Moya Moya disease

DISCUSSION: (Related Text): INCIDENCE The disease is common in japan with an incidence of 3 per $1,00,00$ which is a common cause for paediatric stroke but definitely it is very rare in INDIA. Bi-modal age distribution early childhood: peak $\sim 4$ years of age middle age: 30-40 years of age M:F 1:2 Symptoms at presentation Common Ischemic stroke 50-75 Transient ischemic attack (including drop attacks) 50-75 Hemorrhage (in adults) 10-40 Less common Seizures Headache Suzuki Grading System:

I. Narrowing of ICA apex.

II. Initiation of moyamoya collaterals.

III. Progressive ICA stenosis with intensification of moyamoya-associated collaterals.

IV. Development of ECA collaterals.

V. Intensification of ECA collaterals and reduction of moyamoya-associated vessels.

VI. Total occlusion of ICA and disappearance of moyamoya-associated Collaterals.

JINX about MR angiography: It is safer and easier to perform than conventional angiography. Tends to overestimate the degree of stenosis in the internal carotid artery and other arteries. SURGICAL RX Direct superficial temporal artery-middle cerebral artery anastomosis is not easy to perform in children because both the donor scalp artery and the recipient cortical artery are small. Indirect surgical procedures for moyamoya disease include encephalo myosynangiosis and encephalo duroarterio synangiosis. 
At present, encephalo duroarterio synangiosis is generally accepted as the treatment of choice in children with moyamoya disease Conclusion Moyamoya is a rare disease but should be suspected in unexplained etiology of stroke in both children and adults. Patients with certain conditions such as Down's syndrome and sickle cell disease may be particularly at risk for moyamoya. Characteristic radiographic findings confirm the diagnosis, and recognition of the disease early in its course, with prompt institution of therapy, is critical in order to achieve the best outcome in patients.

Revascularization surgery appears to be effective in preventing stroke in patients with moyamoya. often fails to show fine collateral vessels and, as a result, tends to underestimate moyamoya vessels. Although it has a limitation in accurate preoperative staging, it may have a promising role for evaluating postoperative outcome.

\section{REFERENCES:}

1. O. Togao, F. Mihara from the Department of Clinical Radiology, Graduate School of Medical Sciences, Kyushu University, and the Department of Neurosurgery (T.M.), Yamanomachi Hospital.

2. Fukuoka, Japan Hye-Kyung Yoon Department of Radiology, Samsung Medial Center, Sungkyunkwan University School of Medicine, 50 Irwon-Dong, Kangnam-Gu, Seoul, 135-230, Korea.

3. R. Michael Scott, M.D., and Edward R. Smith, M.D. From the Department of Neurosurgery, Children's Hospital Boston, and Harvard Medical School, Boston.

4. Suzuki J, Kodama N. Moyamoya disease: a review. Stroke 1983;14:104-114

5. Takahashi, Magnification angiography in Moya Moya disease. Radiology, 1980; 136:379-386.

6. Kirkham FJ, De Baun MR. Stroke in children with sickle cell disease. Curr Treat Options Neurol 2004;6:357-75.

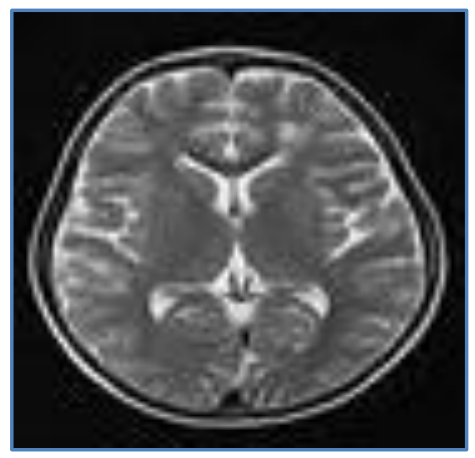

Figure 1: MRI axial

IMAGE 1: MRI: revealed presence of infarction in the bilateral frontal lobes as classic for a paediatric moya moya. (while haemorrhage from collaterals is common in adults). 


\section{CASE REPORT}

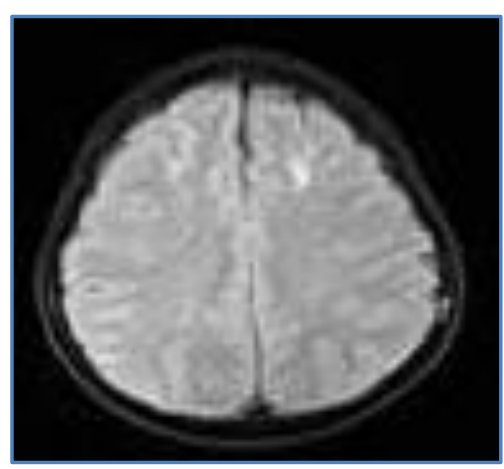

Figure 2: Flair

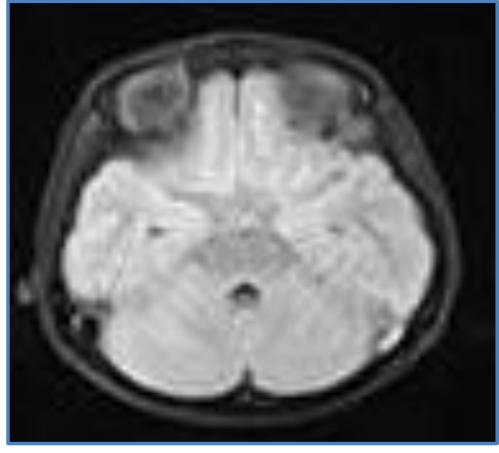

Figure 3: Flair

IMAGE 2 \& 3: Leptomeningeal enhancement the "ivy sign," represents the fine vascular network over the pial surface is demonstrated in FLAIR images.

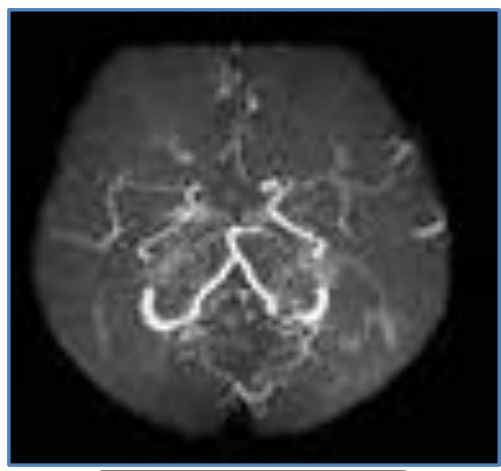

Figure 4: MRA

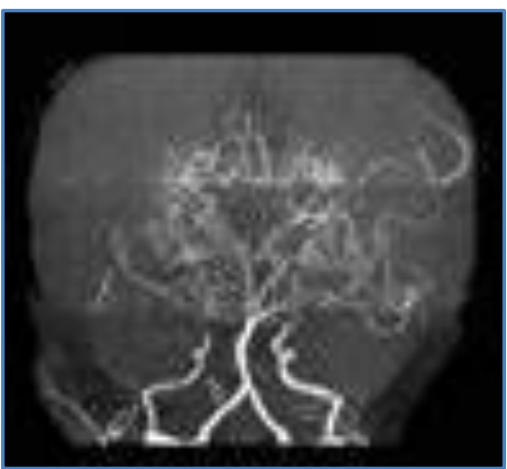

Figure 5: MRA

IMAGE 4 \& 5: MRA: was able to detect abnormal vessels in the basal ganglia, basal cisterns \& periventricular white matter which are known as "MOYA MOYA VESSELS".

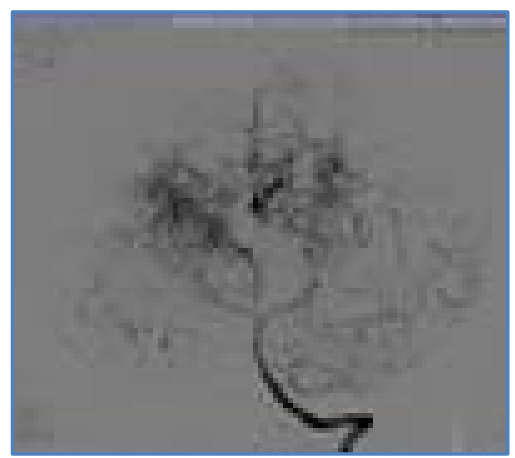

Figure 6: DSA

IMAGE 6: CONVENTIONAL ANGIO (DSA): Occlusion of supra clinoid portion of internal carotid arteries on both sides ( $\mathrm{rt}>\mathrm{lt}$ ) with complete stenosis of M1 segment of rt MCA \& A1 SEGMENT OF RT ACA corresponding to stage IV in rt side \& stage III in left side according to suzuki's classification.

Small abnormal netlike vessels proliferate giving the characteristic "puff of smoke" appearance on direct angiography. 


\section{CASE REPORT}

\section{AUTHORS:}

1. Jai Kishore

2. Ramkumar

3. Bulabai Karpagam

\section{PARTICULARS OF CONTRIBUTORS:}

1. Senior Resident, Department of Radiology, SRM Medical College Hospital and Research Centre.

2. Assistant Professor, Department of Radiology, SRM Medical College Hospital and Research Centre.

3. Associate Professor, Department of Radiology, SRM Medical College Hospital and Research Centre.

\section{NAME ADDRESS EMAIL ID OF THE} CORRESPONDING AUTHOR:

Dr. Bulabai Karpagam, Department of Radiology, SRM Medical College Hospital and Research Centre, Kattankulathur - 603203.

E-mail: karaso1987@yahoo.com

Date of Submission: 01/04/2014.

Date of Peer Review: 02/04/2014.

Date of Acceptance: 18/04/2014.

Date of Publishing: 10/11/2014. 